The extent of atherosclerotic lesions in crural arteries predicts survival of patients with lower limb peripheral artery disease : A new classification of crural atherosclerosis

Jalkanen, Juho M.

2016-08

Jalkanen , J M , Wickstrom , J-E , Venermo , M \& Hakovirta , H H 2016 , ' The extent of atherosclerotic lesions in crural arteries predicts survival of patients with lower limb peripheral artery disease : A new classification of crural atherosclerosis ', Atherosclerosis , vol. 251 , pp. 328-333 . https://doi.org/10.1016/j.atherosclerosis.2016.04.016

http://hdl.handle.net/10138/224537

https://doi.org/10.1016/j.atherosclerosis.2016.04.016

publishedVersion

Downloaded from Helda, University of Helsinki institutional repository.

This is an electronic reprint of the original article.

This reprint may differ from the original in pagination and typographic detail.

Please cite the original version. 


\title{
The extent of atherosclerotic lesions in crural arteries predicts survival of patients with lower limb peripheral artery disease: A new classification of crural atherosclerosis
}

\author{
Juho M. Jalkanen a , Jan-Erik Wickström ${ }^{\text {a }}$, Maarit Venermo ${ }^{\text {b }}$, Harri H. Hakovirta ${ }^{\text {a, * }}$ \\ ${ }^{\text {a }}$ Department of Vascular Surgery, Turku University Hospital and University of Turku, Finland \\ ${ }^{\mathrm{b}}$ Department of Vascular Surgery, University Hospital of Helsinki, Finland
}

\section{A R T I C L E I N F O}

\section{Article history:}

Received 31 January 2016

Received in revised form

4 April 2016

Accepted 19 April 2016

Available online 22 April 2016

\section{Keywords:}

Imaging

Peripheral artery disease

Atherosclerosis

Survival

TASC II classification

\begin{abstract}
A B S T R A C T
Background and aims: Several studies report correlation of ankle brachial index (ABI) values and mortality. However, no studies exist on the predictive value of anatomical distribution of atherosclerotic lesions and the extent of atherosclerosis at defined arterial segments on life expectancy. The aim of the present study was to evaluate the significance of both extent and localisation of atherosclerotic lesions to mid-term patient survival.

Methods: Digital subtraction angiography (DSA) images of 887 consecutive patients admitted to the Department of Vascular Surgery at Turku University Hospital (Turku, Finland) were retrospectively analysed. Each angiography was classified according to the TASC II classification for aorto-iliac and femoro-popliteal segments, and a similar four-grade index was created for crural arteries. Patients were followed until 36-months post DSA.

Results: During 36-month follow-up 295 (33\%) deaths occurred. Death during follow-up was strongly associated with extensive crural disease, but not with extensive proximal disease (Crural Index III-IV, $p=0.044$ and $<0.001$, respectively). In a Cox regression analysis incorporating baseline variables, Crural Index IV and most severe atherosclerosis on crural vessels were the strongest predictors of poor prognosis (HR 2.20 95\% CI 1.3-3.7, $p=0.003$ and HR 2.45 95\% CI 1.5-4.0, $p<0.001$ respectively).

Conclusions: The extent of crural atherosclerosis is independently associated with poor mid term life expectancy. Therefore, a classification of the extent of crural atherosclerosis could serve as an indicator of mortality among PAD patients and aid in clinical decision making.
\end{abstract}

(C) 2016 Elsevier Ireland Ltd. All rights reserved.

\section{Introduction}

Peripheral artery disease (PAD) is an important cause of morbidity and mortality in western countries. PAD-associated

\footnotetext{
List of abbreviations: ABI, Ankle brachial index; AFS, Amputation free survival; CAD, Coronary arterial disease; COPD, Chronic obstructive pulmonary disease; CI, Confidence interval; CVD, Cerebrovascular disease; DSA, Digital subtraction angiography; ERDS, End-stage renal disease; MACCE, Major adverse cardiovascular and cerebrovascular events; PAD, Peripheral arterial disease; SE, Standard error; SD, Standard deviation; TASC, Trans-Atlantic Inter-Society Consensus; WIfl, Wound, Ischemia, and foot Infection.

* Corresponding author. Turku University Hospital, TE5 Hämeentie 11, 20521, Turku, Finland.

E-mail addresses: juho.jalkanen@utu.fi (J.M. Jalkanen), jan-erik.wickstrom@tyks. fi (J.-E. Wickström), maarit.venermo@hus.fi (M. Venermo), haheha@utu.fi (H.H. Hakovirta).
}

disability and death has increased in all age groups during the past 20 years [1]. The life expectancy of patients with diagnosed large vessel PAD has been shown to be shorter than that of patients without diagnosed PAD [2,3]. Furthermore, widespread atherosclerosis of lower extremity arteries is associated with high mortality compared to that of atherosclerosis localised to other vascular beds [4]. Most of these studies are reporting the predictive value of ankle-brachial index (ABI) on mortality. However, the risk of death and its relation to the extent of atherosclerosis in different vascular segments of the lower limb has not been investigated and there are very few clinical scores assessing mortality risk in PAD [5].

No current classification utilises the extent and distribution of lower limb PAD for risk-analysis of PAD patient outcome. The best current descriptive classifications for characterising lesion extent and severity in lower extremity arteries are aimed at invasive treatment of limb ischaemia in the TASC [6] and TASC II [7,8] 
recommendations. Additional classifications of severity and distribution of occlusive arterial lesions in the lower extremity have been characterised $[9,10]$, but none of these have been shown to have predictive value regarding patient outcome. Recently, the runoff score of lower limb arteries has been shown to associate with patient outcome and cardiovascular events [11].

The need for new methods for risk assessment of PAD patients has been emphasised [12]. Earlier observations have strongly suggested that atherosclerosis detected with digital subtraction angiography (DSA) could be a useful marker for patient outcome [13,14]. The aim of the present study was to analyse whether the extent of atherosclerotic lesions in the lower limb arteries has predictive value on the outcome of PAD patients.

\section{Materials and methods}

\subsection{Patient cohort}

The present study is a retrospective study of all 887 consecutive Caucasian patients admitted to the Department of Vascular Surgery at the Turku University Hospital (Turku, Finland) either for diagnostic DSA or for endovascular treatment of PAD from January 1st, 2009 to July 30th, 2011. All patients were included regardless of earlier PAD history. DSA was the gold standard imaging modality to visualize atherosclerotic changes in lower limb arteries at our department during the study period. The DSA from the clinically more severely affected lower limb was analysed. When both limbs met the criteria for critical limb ischaemia, the limb with the lowest toe pressures was considered worse and entered into the study. If a patient had repeated DSAs during the study period, only data from the first period of hospitalisation was analysed. The study cohort consists of both elective and urgent patients. The local Ethical Committee of the Hospital District of South-Western Finland approved this study.

Patient baseline characteristics were retrospectively collected from our electronic hospital database. Only ICD-10 coded diagnoses were registered. The following risk factors were collected for analysis; coronary artery disease (CAD), carotid artery disease, hypertension, active smoking, diabetes, sleep apnoea, chronic obstructive pulmonary disease (COPD), end-stage renal disease (ESRD), dyslipidaemia, ABI, toe pressures, revascularisation modality and medical treatment (anticoagulant, antithrombotic and statin). The Rutherford classification [15] was retrospectively determined from patient files to assess the clinical severity of ischemia. All patient files were followed up to 38 months to determine all-cause mortality and amputation free survival (AFS) as the hospital registry has a delay of up to 2 weeks in updating mortality data. Furthermore, the date and cause of death was provided by the Cause of death registry of Statistics Finland. Deaths within the patient cohort were registered for the first 36 months, which was the cut-off point for follow-up.

\subsection{DSA analysis and description of the crural index}

All DSA images were analysed by the corresponding author. The index classification was as described in TASC II for aorto-iliac and femoro-popliteal segments. Aorto-iliac and femoro-popliteal segments (TASC II classification A-D) were modified as follows; no disease was coded as 0 , TASC II A was coded as 1 , TASC II B was coded as 2 , TASC II $C$ was coded as 3 and TASC II D was coded as 4 for the statistical analyses.

For the crural region, all three vessels were first analysed separately. Each crural vessel was coded as follows: No detectable occlusive disease, or minor stenosis 0 ; Total occlusion less than $5 \mathrm{~cm}$ : 1; Total occlusion less than $10 \mathrm{~cm}$ : 2; Total occlusion less than
$15 \mathrm{~cm}$ : 3; Total occlusion more than $15 \mathrm{~cm}$ : 4 . Only total occlusions were measured, other atherosclerotic lesions were not taken into account. The Crural Index was created by a sum of the three values obtained from each individual crural vessel. If the sum was 0 the Index was 0 , if the sum was between 1 and 3 the Index was I, if the sum was 4-6 the Index was II, if the sum was 7-9 the Index was III, and if the sum was 10-12 the Index was IV.

To assess different vascular segments against each other, each patient was assigned into a specific group of disease localisation: 1) aorto-iliac, 2) femoro-popliteal or 3) crural, based on which 0 - IV rating gave the highest number. For example, according to the TASC II classification, a patient had proximal lesions of grade 0 - II, but a Crural Index of III, the patient was assigned to the crural group. However, if the highest grade was equal in two or even three localisations the patient was assigned to the group of the more proximal lesion. For example, a patient with grade IV lesions in all localisations (aorto-iliac, femoro-popliteal, crural) would have been assigned to the aorto-iliac group.

\subsection{Statistical analyses}

All statistical analyses were performed using the IBM SPSS version 22 statistics program. Categorical variables were expressed as frequency and percentage. For continuous variables, patient characteristics were expressed as mean \pm either standard deviation (SD) or standard error (SE). Normal distribution was assessed with Shapiro-Wilk tests. Survival analyses were assessed by KaplanMeier curves and Log-rank statistics. Survival and death between groups were compared with ANOVA. A Cox regression analysis was performed to assess the final predictive value of factors affecting survival. Factors with $p<0.2$ in univariate analysis were selected as independent variables to the Cox proportional hazard model.

\section{Results}

\subsection{Patient characteristics}

The male-to-female ratio in the study cohort is $57 \%$ and $43 \%$, respectively. During the follow-up time 295 (33\%) deaths were registered. Mean age of the patient cohort was 72.4 years ranging from 40 to 98 years. Baseline characteristics are summarized in Table 1. Crural Index grades 0-IV were compared based on patient characteristics and Rutherford class in Table 2. Patient characteristics by most severely diseased arterial segment are shown in Table 3. ABI measurements were available for 792 patients and toe pressures for 791 patients. Measurements were lacking due to either painful crural wounds, absent toes, gangrene, emergent

Table 1

Baseline characteristics (number of patients) of study cohort Number of patients is 887 in each group except for medication (anticoagulants, antithrombotic and statins) $(\mathrm{n}=825)$.

\begin{tabular}{lc}
\hline Cohort demographics & Prevalence \\
\hline CAD (385) & $43.4 \%$ \\
CVD (147) & $16.6 \%$ \\
Hypertension (618) & $69.7 \%$ \\
Diabetes (379) & $42.7 \%$ \\
Sleep apnoea (51) & $5.7 \%$ \\
ESRD (92) & $10.4 \%$ \\
Dyslipidaemia (310) & $34.9 \%$ \\
Smoking history (237) & $26.7 \%$ \\
COPD (108) & $12.2 \%$ \\
Antithrombotic (599) & $73 \%$ \\
Anticoagulants (198) & $24 \%$ \\
Statins (473) & $57 \%$ \\
\hline
\end{tabular}


Table 2

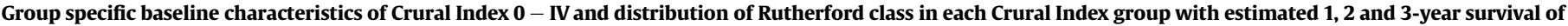

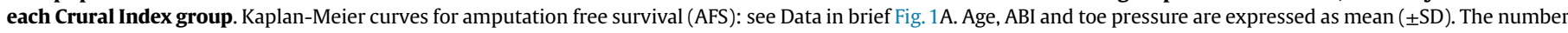

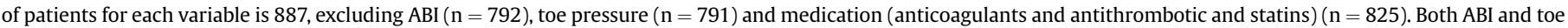
pressure of Crural Index I are significantly higher compared to Crural Index IV ( $p=0.001$ and $p<0.001$, respectively).

\begin{tabular}{|c|c|c|c|c|c|}
\hline Crural Index & Grade 0 & Grade I & Grade II & Grade III & Grade IV \\
\hline $\mathrm{N}$ & 126 & 70 & 235 & 289 & 167 \\
\hline Male/female & $56 / 44 \%$ & $73 / 27 \%$ & $63 / 37 \%$ & $51 / 49 \%$ & $52 / 48 \%$ \\
\hline Age (years) & $66.3( \pm 9.1)$ & $67.9( \pm 9.9)$ & $70.7( \pm 10.2)$ & $74.1( \pm 10.6)$ & $78.7( \pm 10.4)$ \\
\hline CAD & $43 \%$ & $37 \%$ & $39 \%$ & $46 \%$ & $49 \%$ \\
\hline Hypertension & $64 \%$ & $64 \%$ & $69 \%$ & $72 \%$ & $73 \%$ \\
\hline Diabetes & $32 \%$ & $46 \%$ & $44 \%$ & $44 \%$ & $46 \%$ \\
\hline COPD & $18 \%$ & $9 \%$ & $16 \%$ & $11 \%$ & $7 \%$ \\
\hline Smoking history & $57 \%$ & $20 \%$ & $33 \%$ & $16 \%$ & $10 \%$ \\
\hline Sleep apnoea & $7 \%$ & $9 \%$ & $7 \%$ & $5 \%$ & $3 \%$ \\
\hline CVD & $15 \%$ & $14 \%$ & $17 \%$ & $18 \%$ & $16 \%$ \\
\hline ESRD & $6 \%$ & $17 \%$ & $12 \%$ & $12 \%$ & $7 \%$ \\
\hline Antithrombotic & $80 \%$ & $67 \%$ & $78 \%$ & $70 \%$ & $65 \%$ \\
\hline Anticoagulants & $14 \%$ & $27 \%$ & $21 \%$ & $27 \%$ & $31 \%$ \\
\hline Statins & $57 \%$ & $66 \%$ & $57 \%$ & $60 \%$ & $50 \%$ \\
\hline $\mathrm{ABI}$ & $0.56 \pm 0.26$ & $0.83 \pm 0.67$ & $0.66 \pm 0.41$ & $0.67 \pm 0.52$ & $0.54 \pm 0.52$ \\
\hline Toe pressure & $48 \pm 27 \mathrm{mmHg}$ & $50 \pm 26 \mathrm{mmHg}$ & $45 \pm 26 \mathrm{mmHg}$ & $36 \pm 26 \mathrm{mmHg}$ & $27 \pm 24 \mathrm{mmHg}$ \\
\hline Rutherford 1 & $2(1.6 \%)$ & $0(0 \%)$ & $0(0 \%)$ & $0(0 \%)$ & $0(0 \%)$ \\
\hline Rutherford 2 & $3(2.4 \%)$ & $1(1.4 \%)$ & $4(1.7 \%)$ & $0(0 \%)$ & $4(2.4 \%)$ \\
\hline Rutherford 3 & $78(62 \%)$ & $42(60 \%)$ & $99(42 \%)$ & $92(32 \%)$ & $26(16 \%)$ \\
\hline Rutherford 4 & $20(16 \%)$ & $7(10 \%)$ & 45 (19\%) & $68(24 \%)$ & $38(23 \%)$ \\
\hline Rutherford 5 & $15(12 \%)$ & $7(10 \%)$ & $20(8.5 \%)$ & $67(23 \%)$ & $53(32 \%)$ \\
\hline Rutherford 6 & $8(6.3 \%)$ & $13(19 \%)$ & $67(29 \%)$ & $62(21 \%)$ & $46(28 \%)$ \\
\hline Surviving $1,2,3$ years & $87 \%, 83 \%, 83 \%$ & $81 \%, 79 \%, 76 \%$ & $80 \%, 75 \%, 75 \%$ & $71 \%, 65 \%, 63 \%$ & $55 \%, 44 \%, 42 \%$ \\
\hline AFS $1,2,3$ years & $87,81 \%, 79 \%$ & $77 \%, 76,73 \%$ & $79 \%, 74 \%, 74 \%$ & $67 \%, 60 \%, 58 \%$ & $49 \%, 40 \%, 37 \%$ \\
\hline
\end{tabular}

Table 3

Group specific baseline characteristics by segment with most severely diseased arterial segment. Amputation free survival (AFS). Kaplan-Meier curves for AFS: see Data in brief Fig. 1B. Age, ABI and toe pressure are expressed as mean $( \pm \mathrm{SD})$. The number of patients for each variable is 887 , excluding $A B I(n=792)$, toe pressure $(\mathrm{n}=791)$ and medication (anticoagulants and antithrombotic and statins) $(\mathrm{n}=825)$. The ABI and toe pressures in crural group are significantly $(p<0.001)$ lower compared to both aorto-iliac and femoro-popliteal groups.

\begin{tabular}{llll}
\hline Most severe segment & Aorto-iliac & Femoro-popliteal & Crural \\
\hline Patients & 141 & 416 & 325 \\
Male/female & $65 / 35 \%$ & $58 / 42 \%$ & $51 / 49 \%$ \\
Age (years) & $66.7( \pm 10.1)$ & $71.8( \pm 10.1)$ & $76.0( \pm 10.7)$ \\
CAD & $42 \%$ & $44 \%$ & $44 \%$ \\
Hypertension & $67 \%$ & $69 \%$ & $71 \%$ \\
Diabetes & $23 \%$ & $41 \%$ & $53 \%$ \\
COPD & $18 \%$ & $16 \%$ & $5 \%$ \\
Smoking history & $52 \%$ & $32 \%$ & $9 \%$ \\
Sleep apnea & $6 \%$ & $6 \%$ & $6 \%$ \\
CVD & $14 \%$ & $17 \%$ & $17 \%$ \\
ESRD & $6 \%$ & $8 \%$ & $15 \%$ \\
Antithrombotic & $83 \%$ & $74 \%$ & $67 \%$ \\
Anticoagulants & $15 \%$ & $24 \%$ & $28 \%$ \\
Statins & $62 \%$ & $58 \%$ & $54 \%$ \\
ABI & $0.54 \pm 0.22$ & $0.60 \pm 0.45$ & $0.75 \pm 59$ \\
Toe pressure & $47 \pm 27 \mathrm{mmHg}$ & $43 \pm 27 \mathrm{mmHg}$ & $33 \pm 22 \mathrm{mmHg}$ \\
Rutherford 1 & $0 \%$ & $100 \%$ & $0 \%$ \\
Rutherford 2 & $8 \%$ & $58 \%$ & $33 \%$ \\
Rutherford 3 & $16 \%$ & $58 \%$ & $26 \%$ \\
Rutherford 4 & $15 \%$ & $52 \%$ & $32 \%$ \\
Rutherford 5 & $8 \% \%$ & $33 \%$ & $59 \%$ \\
Rutherford 6 & $6 \%$ & $35 \%$ & $60 \%$ \\
Surviving 1,2,3 years & $89 \%, 83 \%, 82 \%$ & $83 \%, 78 \%, 77 \%$ & $56 \%, 47 \%, 45 \%$ \\
AFS 1,2,3 years & $86 \%, 79 \%, 78 \%$ & $80 \%, 75 \%, 74 \%$ & $51 \%, 44 \%, 41 \%$ \\
\hline
\end{tabular}

revascularisation or non-compliance with the treatment protocol. Medication history could be reliably collected from electronic patient files for 825 patients. The electronic medication record was introduced during the study period and medication status could not be reliably determined for 62 patients.

\subsection{Survival and extent of atherosclerosis}

A four-grade classification was utilised for survival analyses. Segments were graded as described in the methods section. Within the aorto-iliac segment significant differences between grades I and II ( $p=0.015$ and $p=0.024$ respectively) compared to grade III were detected (Data in brief, Table 2A). No significant differences in survival were detected between different grades of femoropopliteal disease (Data in brief, Table 2B).

Crural Index III was found to show significantly shorter estimated survival compared to grades I and II ( $p=0.044$ and $p=0.004$, respectively) and crural Index IV was found to show significantly shorter estimated survival compared to grades I and II ( $p<0.001$ and $p<0.001$, respectively). In addition, estimated survival in grade IV crural disease differed significantly from that of grade III $(p<0.001$, Data in brief Table $2 C)$. The Kaplan-Meier curves of Crural Index grades I-IV are illustrated in Fig. 1A (Data in Brief Table 2C). The Kaplan-Meier curves of Crural Index grades I-IV and AFS are illustrated in Data in brief, Fig. $1 \mathrm{~A}$ and Table $1 \mathrm{C}$. The cause of death was cardiovascular in $70 \%$ of cases with crural index III and $67 \%$ with crural index IV.

DSA data was also analysed by segments with significant atherosclerotic lesions on a yes/no basis. Patients with significant atherosclerotic lesions localised only to the crural arteries had a significantly shorter estimated survival compared to those with disease localisation to the aorto-iliac $(p<0.001)$ or femoropopliteal $(p=0.011)$ segments. In addition, disease localisation to both aorto-iliac and femoro-popliteal segments demonstrated significantly longer survival compared to isolated crural disease $(p=0.014)$. Furthermore, isolated crural disease resulted in significantly $(p=0.011)$ shorter estimated survival than disease localised to both aorto-iliac and crural segments. When all three segments had significant atherosclerotic lesions, or the significant atherosclerotic lesions were localised to femoro-popliteal and crural segments, the estimated mean survival was significantly $(P=0.011)$ shorter compared to aorto-iliac disease (Data in Brief, Table 2D). 

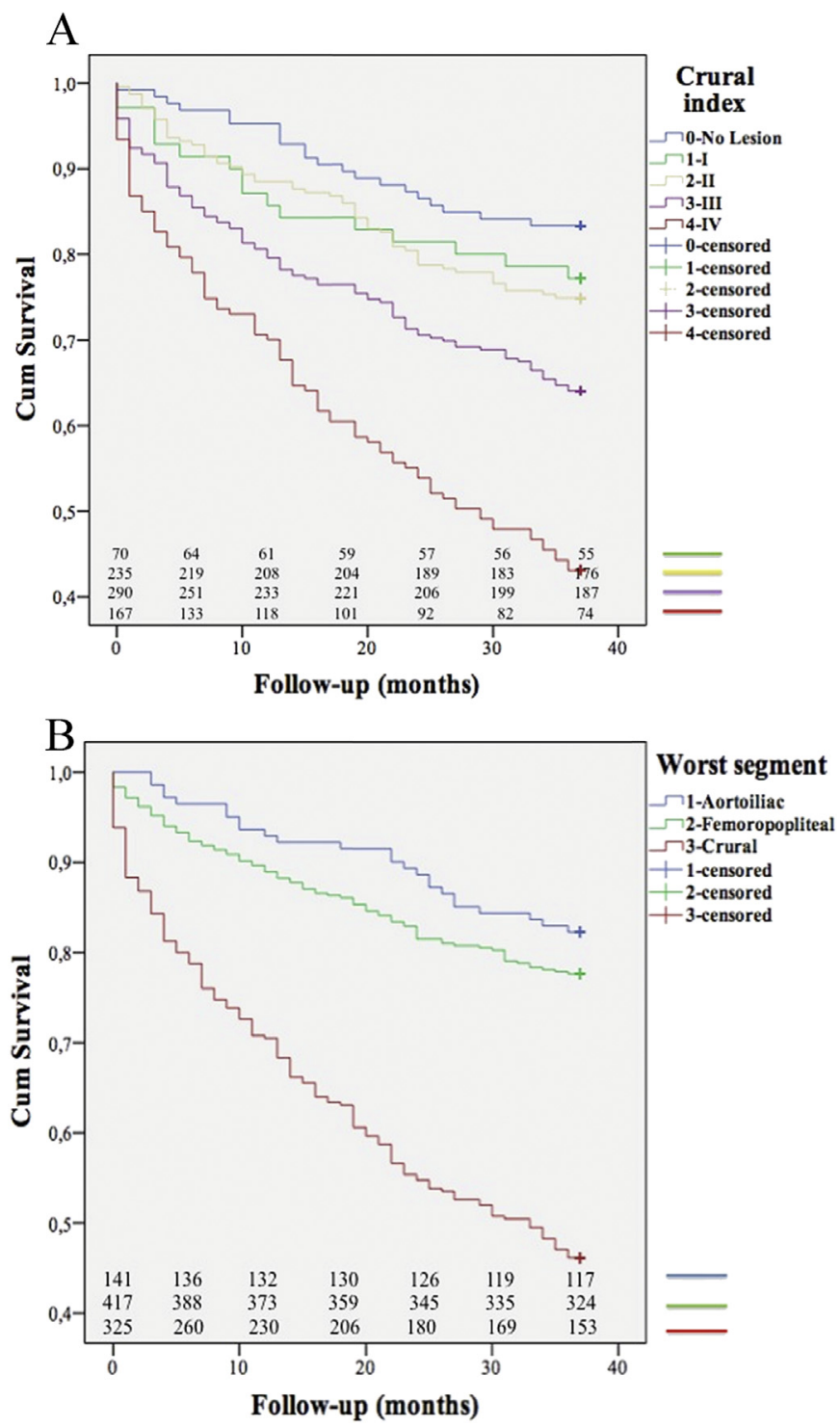

Fig. 1. Kaplan-Meier curves. (A) Kaplan-Meier curves show the cumulative survival during 36-month follow-up. Separate curves for Crural Index I-IV and for patients with no detectable significant atherosclerotic lesion in crural arterial vessels. The survival curve of Crural Index IV demonstrates poor prognosis for patients with extensive atherosclerosis in crural arteries. Numbers at risk for each curve marked at defined time-point. Corresponding Kaplan-Meier curves for amputation free survival (AFS): see Data in brief Fig. 1A. (B) Kaplan-Meier curves demonstrating survival based on most severely diseased vascular segments. A more detailed presentation of data analysis is given in the Materials and Methods section. The segments are marked as aorto-iliac (AI), femoro-popliteal (FP) and crural ( $\mathrm{Cr}$ ). Severe crural lesions predict a poor prognosis. Numbers at risk for each curve marked at defined time-point. Corresponding Kaplan-Meier curves for AFS: see Data in brief Fig. 1B.

Analysis of most severely diseased arterial segment revealed that the cohort suffering from severe crural disease had a significantly ( $p<0.001$ ) shorter mean estimated survival compared to the groups with femoro-popliteal or aorto-iliac disease (Data in Brief, Table 2E). The Kaplan-Meier curves of most severely diseased segment and survival are illustrated in Fig. 1B (AFS in Data in brief, Fig. 1B).

\subsection{Predictive value of factors affecting survival}

A Cox regression analysis was run to assess the predictive value of risk factors, ABI and Crural Index affecting survival in the study.
Cardiovascular risk factors diabetes (HR 1.42; 95\% CI, 1.1-1.8; $p=0.015$ ) and end-stage renal disease (HR 2.09; 95\% CI, 1.4-3.0; $p<0.001)$ and ABI (HR 1.62; 95\% CI, 1.3-2.1; $p<0.001)$ associated with a significantly higher risk of death. However, the strongest predictor of death was Crural Index IV (HR 2.20; 95\% CI, 1.3-3.7; $p=0.003$ ) (Table 4A).

The Cox regression analysis was also run to assess the predictive value of risk factors, $\mathrm{ABI}$ and most diseased segment of the lower limb arteries. The strongest predictor was severe crural disease (HR 2.45; 95\% CI, 1.5-4.0; $p<0.001$ ). Diabetes (HR 1.33; 95\% CI, 1.0-1.7; $p=0.043$ ) and end-stage renal disease (HR 1.81; 95\% CI, 1.2-2.6; $p=0.002$ ), but not $A B I$, were associated with a significantly higher risk of death (Table $4 \mathrm{~B}$ ).

\section{Discussion}

Previous literature has shown that significant atherosclerotic lesions at aorto-iliac or femoro-popliteal segments have been associated with death [13]. In addition, the risk of major adverse cardiovascular and cerebrovascular events (MACCE) has been shown to be associated with significant atherosclerotic lesions (>50\% stenosis) at the aorto-iliac segment, but not crural atherosclerosis [14]. An opposite observation has been reported in an extensive study utilising non-invasive screening of atherosclerosis at three segments of lower limb vascular beds, demonstrating that distal atherosclerosis is prognostic for poor survival [16]. However, none of these studies which have shown correlation between the level of lower limb atherosclerosis and survival have focused on the possible predictive value of both extent and distribution of atherosclerotic lesions of the lower extremity on patient survival.

Several different classifications of lower extremity PAD using angiographic analysis have been established [7-10], but these

\section{Table 4}

Cox regression analysis. (A) Cox regression analysis assessing the predictive value of risk factors and the Crural Index (Fig. 1A) affecting survival during 36-month follow-up. (B) Cox regression analysis assessing the predictive value of risk factors and most severe atherosclerosis on crural vessels (Fig. 1B) affecting survival during 36-month follow-up.

\begin{tabular}{|c|c|c|c|}
\hline & Hazard ratio & $95.0 \% \mathrm{CI}$ & $p$ Value \\
\hline \multicolumn{4}{|l|}{$\bar{A}$} \\
\hline Age & 1.04 & $1.0-1.1$ & $<0.001$ \\
\hline Crural Index I & 1.04 & $0.5-2.1$ & 0.905 \\
\hline Crural Index II & 1.05 & $0.63-1.8$ & 0.846 \\
\hline Crural Index III & 1.36 & $0.82-2.2$ & 0.237 \\
\hline Crural Index IV & 2.20 & $1.3-3.7$ & 0.003 \\
\hline $\mathrm{ABI}$ & 1.62 & $1.3-2.1$ & $<0.001$ \\
\hline Toe pressure & 0.989 & $0.98-0.99$ & $<0.001$ \\
\hline CAD & 1.00 & $0.92-1.1$ & 0.984 \\
\hline CVD & 0.521 & $0.34-0.79$ & 0.002 \\
\hline DM & 1.42 & $1.1-1.8$ & 0.015 \\
\hline COPD & 1.19 & $0.79-1.8$ & 0.411 \\
\hline ESRD & 2.09 & $1.4-3.0$ & $<0.001$ \\
\hline Smoking history & 0.638 & $0.43-0.95$ & 0.028 \\
\hline Dyslipidaemia & 0.890 & $0.65-1.2$ & 0.352 \\
\hline \multicolumn{4}{|l|}{ B } \\
\hline$\overline{\text { Age }}$ & 1.04 & $1.0-1.1$ & $<0.001$ \\
\hline Crural & 2.45 & $1.5-4.0$ & $<0.001$ \\
\hline $\mathrm{ABI}$ & 1.36 & $1.1-1.7$ & 0.019 \\
\hline Toe pressure & 0.990 & $0.98-1.0$ & $<0.001$ \\
\hline CAD & 1.01 & $0.93-1.1$ & 0.756 \\
\hline CVD & 0.496 & $0.33-0.76$ & 0.001 \\
\hline DM & 1.33 & $1.0-1.7$ & 0.043 \\
\hline COPD & 1.43 & $0.95-2.2$ & 0.091 \\
\hline ESRD & 1.81 & $1.2-2.6$ & 0.002 \\
\hline Smoking history & 0.697 & $0.47-1.0$ & 0.077 \\
\hline Dyslipidaemia & 0.973 & $0.73-1.3$ & 0.850 \\
\hline
\end{tabular}


classifications aim to guide the choice of lower limb PAD treatment modality or to classify the severity of atherosclerosis. Characterised in the present study, the Crural Index is the first classification based on grading the extent of atherosclerosis detectable utilising DSA that has predictive value on mid-term survival of lower limb PAD patients. In accordance with our results, there is a previous observation that PAD patients with isolated crural disease have poorer life expectancy than patients with multilevel disease, even when the multilevel disease involves the crural arteries [17]. Unfortunately, the extent of atherosclerotic lesions is not analysed in the study by Gray et al. Similarly, isolated tibial disease has been associated with worse outcomes after revascularisation when compared to a multilevel disease [18]. These findings together with observations that poor runoff is predictive of poor patient survival [11] are in accordance with our observations. Whether the newly applied classification (WIfI) for severity of ischaemic ulcers will be predictive for patient survival, remains an interesting target for future research $[19,20]$.

Earlier studies utilising DSA images to analyse atherosclerosis in the lower extremities have shown that aorto-iliac lesions are associated with poor outcome measured as MACCE and death [14]. Additionally, aorto-iliac calcification detected by computed tomography has been shown to be associated with increased mortality [4]. Contrary to these earlier segment-specific observations, we could not confirm the negative prognostic value of aorto-iliac disease on patient outcome using the TASC II classification as a means to measure the extent of atherosclerosis. This controversy between previous observations and present data could be explained by the difference in grading the disease in the vascular segments of the lower limb. The aim of the TASC II classification is essentially to guide the choice of treatment modality, whereas earlier studies utilised simply detecting the presence, but not the extent, of significant atherosclerotic lesions [14]. Outcome was furthermore measured by MACCE as well as survival [14], which may explain differences in the results of these two studies. In our analysis, the extent of atherosclerosis in crural arteries was significantly associated with poor outcome. Unlike single atherosclerotic lesion assessment on a yes/no basis, Crural Index grades III and IV are indicators of widespread atherosclerotic lesions in crural arteries. Less extensive lesions, as measured by the Crural Index, demonstrate markedly better survival. This clear difference in survival between patients with lesions of differing significance in the crural arteries may have been lost, and the poor outcome therefore not detected [21]. Further supporting present findings, an extensive study utilising non-invasive diagnostic ultrasound screening of atherosclerosis at various lower limb vascular beds, demonstrated that atherosclerosis in crural arteries has independent prognostic value for poor survival of a PAD patients [16]. Together with our data these observations further emphasise the role of severe extensive crural atherosclerosis as a potent marker of poor survival, with $57 \%$ mortality at 36 -month follow-up.

Co-morbidities and risk factors in the present study correlated essentially with risk factors demonstrated in earlier studies concerning lower limb PAD patients [2,22-24]. Smoking is associated with femoro-popliteal [25] and aorto-iliac atherosclerosis [26]. The extent of proximal atherosclerosis was not found to be associated with poor survival in our study. The prevalence of a history of smoking was very low in subjects with extensive crural atherosclerosis. Thus, smoking independently is likely a major contributing factor of death amongst a defined group of younger subjects with extensive proximal atherosclerosis. The better survival of patients with diagnosed carotid artery disease emphasises the importance of adequate secondary prevention in obtaining optimal survival of PAD patients [27]. In accordance with previous studies, risk of death is clearly associated with diabetes, end-stage renal disease and ABI, which were more prevalent in subjects with extensive crural atherosclerosis. Despite this, the strongest independent predictor of death in the present study was severe crural atherosclerosis.

End-stage renal disease and diabetes are associated with crural PAD [28-31]. An ankle brachial index demonstrating mediasclerosis together with occlusive PAD has been shown to be associated with poor patient survival [32]. These risk factors, diabetes and end-stage renal failure together with critical ischaemia, are shown to be associated with a poor prognosis [17] and they correlate with the extent of atherosclerosis in crural vessels. As expected, the clinical presentation of ischaemia measured by the Rutherford classification correlated with the extent of atherosclerosis in crural arteries resulting in poor distal run-off and insufficient tissue perfusion. The distribution of Rutherford classes 4-6 (rest pain to severe tissue loss) was relatively evenly distributed in Crural Index groups III and IV (Table 2).

Although one of the aims of the study was to detect a possible correlation with the TASC II classification and survival, this was not accomplished. The present study shows for the first time the predictive value of the classification of the extent of arterial lesions in crural vessels on PAD patient survival. The Crural Index is feasible in everyday vascular practice and is a good indicator of patient outcome. It is applicable for assessing patient mid-term survival, which may be crucial when making decisions on extensive, highrisk operative or endovascular revascularisations.

\section{Conflict of interest}

The authors declared that they do not have anything to disclose regarding conflict of interest with respect to this manuscript.

\section{Financial support}

This study was supported by an EVO grant (grant number 13605), Turku University Hospital.

\section{References}

[1] U.K.A. Sampson, P.E. Norman, F.G.R. Fowkes, V. Aboyans, Yanna Song, F.E. Harrell, et al., Global and regional burden of aortic dissection and aneurysms: mortality trends in 21 world regions, 1990 to 2010, Glob. Heart 9 (2014) 171-180, http://dx.doi.org/10.1016/j.gheart.2013.12.010 e10.

[2] M.H. Criqui, R.D. Langer, A. Fronek, H.S. Feigelson, M.R. Klauber, T.J. McCann, et al., Mortality over a period of 10 years in patients with peripheral arterial disease, N. Engl. J. Med. 326 (1992) 381-386, http://dx.doi.org/10.1056/ NEJM199202063260605.

[3] B.A. Golomb, T.T. Dang, M.H. Criqui, Peripheral arterial disease: morbidity and mortality implications, Circulation 114 (2006) 688-699, http://dx.doi.org/ 10.1161/CIRCULATIONAHA.105.593442.

[4] M.A. Allison, S. Hsi, C.L. Wassel, C. Morgan, J.H. Ix, C.M. Wright, et al., Calcified atherosclerosis in different vascular beds and the risk of mortality, Arterioscler. Thromb. Vasc. Biol. 32 (2012) 140-146, http://dx.doi.org/10.1161/ ATVBAHA.111.235234.

[5] G. Hackl, K. Belaj, T. Gary, P. Rief, H. Deutschmann, G. Seinost, et al., COPART risk score predicts long-term mortality in peripheral arterial occlusive disease, Eur. J. Vasc. Endovasc. Surg. 50 (2015) 94-100, http://dx.doi.org/10.1016/ j.ejvs.2015.04.009.

[6] J.A. Dormandy, R.B. Rutherford, Management of peripheral arterial disease (PAD). TASC working group. TransAtlantic Inter-Society Consensus (TASC), J. Vasc. Surg. (2000) S1-S296.

[7] L. Norgren, W.R. Hiatt, J.A. Dormandy, M.R. Nehler, K.A. Harris, F.G.R. Fowkes et al., Inter-society consensus for the management of peripheral arterial disease (TASC II), J. Vasc. Surg. (2007) S5-S67, http://dx.doi.org/10.1016/ j.jvs.2006.12.037. Elsevier.

[8] L. Norgren, W.R. Hiatt, J.A. Dormandy, M.R. Nehler, K.A. Harris, F.G.R. Fowkes, Inter-society consensus for the management of peripheral arterial disease (TASC II), Eur. J. Vasc. Endovasc. Surg. 33 (2007) S1-S75, http://dx.doi.org/ 10.1016/j.ejvs.2006.09.024.

[9] A. Bollinger, K. Breddin, H. Hess, F.M. Heystraten, J. Kollath, A. Konttila, et al. Semiquantitative assessment of lower limb atherosclerosis from routine angiographic images, Atherosclerosis 38 (1981) 339-346.

[10] L. Graziani, A. Silvestro, V. Bertone, E. Manara, R. Andreini, A. Sigala, et al., 
Vascular involvement in diabetic subjects with ischemic foot ulcer: a new morphologic categorization of disease severity, Eur. J. Vasc. Endovasc. Surg. 33 (2007) 453-460, http://dx.doi.org/10.1016/j.ejvs.2006.11.022.

[11] W.S. Jones, M.R. Patel, T.T. Tsai, A.S. Go, R. Gupta, N. Hedayati, et al., Anatomic runoff score predicts cardiovascular outcomes in patients with lower extremity peripheral artery disease undergoing revascularization, Am. Heart J. 170 (2015) 400-408, http://dx.doi.org/10.1016/j.ahj.2015.04.026.

[12] J. Chung, J.G. Modrall, R.J. Valentine, The need for improved risk stratification in chronic critical limb ischemia, J. Vasc. Surg. 60 (2014) 1677-1685, http:// dx.doi.org/10.1016/j.jvs.2014.07.104.

[13] M.T. Vogt, S.K. Wolfson, L.H. Kuller, Segmental arterial disease in the lower extremities: correlates of disease and relationship to mortality, J. Clin. Epidemiol. 46 (1993) 1267-1276, http://dx.doi.org/10.1016/0895-4356(93) 90091-E.

[14] V. Aboyans, I. Desormais, P. Lacroix, J. Salazar, M.H. Criqui, M. Laskar, The general prognosis of patients with peripheral arterial disease differs according to the disease localization, J. Am. Coll. Cardiol. 55 (2010) 898-903, http:/ dx.doi.org/10.1016/j.jacc.2009.09.055.

[15] R.B. Rutherford, J.D. Baker, C. Ernst, K.W. Johnston, J.M. Porter, S. Ahn, et al., Recommended standards for reports dealing with lower extremity ischemia: revised version, J. Vasc. Surg. 26 (1997) 517-538.

[16] Q. Chen, C.Y. Smith, K.R. Bailey, P.W. Wennberg, I.J. Kullo, Disease location is associated with survival in patients with peripheral arterial disease, J. Am. Heart Assoc. 2 (2013), http://dx.doi.org/10.1161/JAHA.113.000304 e000304-e000304.

[17] B.H. Gray, A.A. Grant, C.A. Kalbaugh, D.W. Blackhurst, E.M. Langan, S.A. Taylor, et al., The impact of isolated tibial disease on outcomes in the critical limb ischemic population, Ann. Vasc. Surg. 24 (2010) 349-359, http://dx.doi.org/ 10.1016/j.avsg.2009.07.034.

[18] N. Fernandez, R. McEnaney, L.K. Marone, R.Y. Rhee, S. Leers, M. Makaroun, et al., Multilevel versus isolated endovascular tibial interventions for critical limb ischemia, J. Vasc. Surg. 54 (2011) 722-729, http://dx.doi.org/10.1016/ j.jvs.2011.03.232.

[19] D.L. Cull, G. Manos, M.C. Hartley, S.M. Taylor, E.M. Langan, J.F. Eidt, et al., An early validation of the society for vascular surgery lower extremity threatened limb classification system, J. Vasc. Surg. 60 (2014) 1535-1541, http:// dx.doi.org/10.1016/j.jvs.2014.08.107.

[20] L.X. Zhan, B.C. Branco, D.G. Armstrong, J.L. Mills, The Society for Vascular Surgery lower extremity threatened limb classification system based on Wound, Ischemia, and foot Infection (WIfl) correlates with risk of major amputation and time to wound healing, J. Vasc. Surg. 61 (2015) 939-944 http://dx.doi.org/10.1016/j.jvs.2014.11.045.

[21] V. Aboyans, I. Desormais, P. Lacroix, J. Salazar, M.H. Criqui, M. Laskar, The general prognosis of patients with peripheral arterial disease differs according to the disease localization, Jac 55 (2010) 898-903, http://dx.doi.org/10.1016/ j.jacc.2009.09.055.

[22] C. Kügler, G. Rudofsky, The role of comorbidity burden for patients with symptomatic peripheral arterial disease (PAD). A quantitative approach, Int Angiol. 22 (2003) 290-301.

[23] V. Suominen, T. Rantanen, M. Venermo, J. Saarinen, J. Salenius, Prevalence and risk factors of PAD among patients with elevated ABI, Eur. J. Vasc. Endovasc. Surg. 35 (2008) 709-714, http://dx.doi.org/10.1016/j.ejvs.2008.01.013.

[24] F. Marsico, D. Ruggiero, A. Parente, E. Pirozzi, F. Musella, F. Lo Iudice, et al., Prevalence and severity of asymptomatic coronary and carotid artery disease in patients with lower limbs arterial disease, Atherosclerosis 228 (2013) 386-389, http://dx.doi.org/10.1016/j.atherosclerosis.2013.03.025.

[25] F.B. Smith, A.J. Lee, F.G.R. Fowkes, G.D.O. Lowe, A. Rumley, Variation in cardiovascular risk factors by angiographic site of lower limb atherosclerosis, Eur. J. Vasc. Endovasc. Surg. 11 (1996) 340-346, http://dx.doi.org/10.1016/S10785884(96)80082-7.

[26] N. Diehm, A. Shang, A. Silvestro, D.-D. Do, F. Dick, J. Schmidli, et al., Association of cardiovascular risk factors with pattern of lower limb atherosclerosis in 2659 patients undergoing angioplasty, Eur. J. Vasc. Endovasc. Surg. 31 (2006) 59-63, http://dx.doi.org/10.1016/j.ejvs.2005.09.006.

[27] A.T. Cohen, S. Imfeld, J. Markham, S. Granziera, The use of aspirin for primary and secondary prevention in venous thromboembolism and other cardiovascular disorders, Thromb. Res. 135 (2015) 217-225, http://dx.doi.org/ 10.1016/j.thromres.2014.11.036.

[28] N. Diehm, S. Rohrer, I. Baumgartner, H. Keo, D. Do, C. Kalka, Distribution pattern of infrageniculate arterial obstructions in patients with diabetes mellitus and renal insufficiency - implications for revascularization, Vasa 37 (2008) 265-273, http://dx.doi.org/10.1024/0301-1526.37.3.265.

[29] S. Wasmuth, I. Baumgartner, D.-D. Do, T. Willenberg, A. Saguner, M. Zwahlen, et al., Renal insufficiency is independently associated with a distal distribution pattern of symptomatic lower-limb atherosclerosis, Eur. J. Vasc. Endovasc. Surg. 39 (2010) 591-596, http://dx.doi.org/10.1016/j.ejvs.2009.11.034.

[30] M. Haltmayer, T. Mueller, W. Horvath, C. Luft, W. Poelz, D. Haidinger, Impact of atherosclerotic risk factors on the anatomical distribution of peripheral arterial disease, Int. Angiol. 20 (2001) 200-207.

[31] X. Guo, Y. Shi, X. Huang, M. Ye, G. Xue, J. Zhang, Features analysis of lower extremity arterial lesions in 162 diabetes patients, J. Diabetes Res. 2013 (2013) 781360-781365, http://dx.doi.org/10.1155/2013/781360.

[32] V. Aboyans, P. Lacroix, M.-H. Tran, C. Salamagne, S. Galinat, F. Archambeaud, et al., The prognosis of diabetic patients with high ankle-brachial index depends on the coexistence of occlusive peripheral artery disease, J. Vasc. Surg. 53 (2011) 984-991, http://dx.doi.org/10.1016/j.jvs.2010.10.054. 